\title{
A la mémoire de Judith McA'Nulty \\ Professeure au département de linguistique de l'Université du Québec à Montréal
}

Judith McA'Nulty est morte le 14 septembre 1984, à la suite d'un cancer qu'elle a assumé courageusement pendant deux années et qui ne l'a pas empêchée de poursuivre ses activités de recherche et d'enseignement. Nous voulons ici brièvement mettre en relief les étapes de sa carrière et de sa contribution à la linguistique.

Après une scolarité de maîtrise en littérature et une maîtrise en linguistique sur la dérivation du groupe verbal français, Judith commençait sa carrière à l'UQAM comme professeur régulier en juillet 1970. Durant toute sa carrière, ses charges de cours furent surtout celles de syntaxe, de sémantique et de lexicologie. Ses cours, qu'elle préparait toujours avec grand soin, présentaient fidèlement l'état actuel des connaissances et exigeaient beaucoup de ses étudiants. Elle a su susciter chez plusieurs l'intérêt pour le domaine. Sa recherche a toujours été liée à des problèmes théoriques en grammaire générative, en général, sur la base des données du français. Parmi ses articles, qui présentaient des critiques de certaines positions centrales à la théorie du mouvement et qui se sont trouvées justifiées par la suite, ou qui introduisaient des idées nouvelles, souvent proposées indépendamment ultérieurement, comme l'idée de définir la c-commande en terme de projection maximale ou encore l'idée de traiter INFL comme la tête de $\mathrm{S}$, mentionnons "Les transformations d'extraction et les contraintes de Chomsky", "Syntaxe et sémantique du français", Cahiers de linguistique no 8, PUQ, 1978; "Binding without Case", NELS X, Cahiers de linguistique d'Ottawa, no 9, 1980; "MultiLevel Binding", NELS XIII, GLSA, UMass at Amherst, 1983; "A' Binding", Cornell Working Papers in Linguistics, no. 4, 1983. Ces deux dernières années, elle dirigeait un projet de recherche important subventionné par le Conseil de recherches en sciences humaines du Canada qui visait à élaborer une théorie 
du liage qui permettait de rendre compte de manière élégante de constructions particulières aux langues romanes, notamment les structures à clitiques, les causatives, les quantificateurs flottants, les sujets nuls. Sa manière de considérer la linguistique s'harmonisait bien avec la tendance actuelle de concevoir les caractéristiques des langues naturelles comme le résultat de l'interaction de principes appartenant à divers composantes de la grammaire.

Outre ses tâches d'enseignement et de recherche, elle s'est montrée généreuse de son temps en s'occupant de multiples tâches académiques et liées à l'édition, à l'intérieur et à l'extérieur de sa propre université, en tant que rédactrice des Cahiers de linguistique de l'Université du Québec, puis directrice de la Revue Québécoise de linguistique. Elle faisait partie de plusieurs comités pour l'aide à la recherche, notamment de la Fédération canadienne des études humaines (CCH) et du Programme d'aide financière aux chercheurs et aux créateurs (PAFAC). Elle a également organisé des congrès de linguistique, dont le NELS XIII et elle a collaboré a l'organisation de la section de linguistique du 50e congrès de l'ACFAS.

Son intelligence, son humilité et sa gentillesse lui avaient attiré le respect et l'amitié de très nombreux collègues surtout au Canada, en France et aux Etats-Unis. Nul doute que son souvenir restera longtemps avec eux.

Paul Hirschbühler

Universite d'Ottawa

Anne-Marie di Sciullo

Université du Québec a Montréal 